\title{
Research progresses in roles of LncRNA and its relationships with breast cancer
}

\author{
Xu Bin ${ }^{1+}$, Yang Hongjian ${ }^{2+}$, Zhang Xiping ${ }^{2 *}\left(\mathbb{0}\right.$, Chen $\mathrm{Bo}^{3}$, Yang Shifeng ${ }^{3}$ and Tang Binbin ${ }^{4}$
}

\begin{abstract}
Some progresses have been made in research of long non-coding RNA (hereunder referred to as LncRNA) related to breast cancer. Lots of data about LncRNA transcription concerning breast cancer have been obtained from large-scale omics research (e.g. transcriptomes and chips). Some LncRNAs would become indices for detecting breast cancer and judging its development and prognosis. LncRNAs may affect genesis and development of breast cancer in multiple ways. Perhaps they could develop into potential targets for treating breast cancer if they are carcinogenic. Like those from other studies of breast cancer, many data gained from omics research remain to be validated by much experimental work. For instance, it is still necessary to demonstrate reliability of LncRNAs as indices for diagnosing breast cancer and judging its prognosis (particularly for various subtypes of breast cancer), effectiveness and feasibility of these genes for treating breast cancer as targets. In this paper, recent years'literatures about LncRNAs which are related to breast cancer are summarized and sorted out to review the research progresses in relationships between LncRNAs and breast cancer.
\end{abstract}

Keywords: LncRNA, Breast cancer, Promote, Inhibit, Target

\section{Introduction}

As a type of non-coding RNAs with over 200 nucleotides, LncRNAs may regulate physiological functions of organisms from the perspectives of epigenetics, transcription and post-transcription. Some of them are discovered to be involved in some important processes of breast cancer, including genesis, development, drug resistance and metastasis of breast cancer (BC), while some others may inhibit these processes. No matter they promote or inhibit the processes of breast cancer, their common mechanism of action consists in that they impact proliferation, apoptosis, drug resistance or invasion of $\mathrm{BC}$ cells. Some attempts are being made to develop some LncRNAs into targets for treating $\mathrm{BC}$, and biomarkers for diagnosing $\mathrm{BC}$, judging its prognosis or predicting metastasis and survival. In this paper, these years' related literatures are collected, sorted out and summarized

\footnotetext{
*Correspondence: zxp99688@sina.com

${ }^{+}$Xu Bin and Yang Hongjian contributed equally to this work

${ }^{2}$ Department of Breast Surgery, Zhejiang Cancer Hospital, Banshanqiao,

No. 38 Guangji Road, Hangzhou 310022, Zhejiang, China

Full list of author information is available at the end of the article
}

to review research progresses in relationships between LncRNAs and BC.

\begin{abstract}
A brief introduction to LncRNAs
Genomes of eukaryotes may transcript several types of RNAs, including protein-coding mRNAs, short and long non-coding RNAs (LncRNAs) [1]. From these RNAs, people have discovered that there are more non-coding RNAs than coding RNAs in human cells. According to encyclopedia of DNA elements (ENCODE), 76\% human genomic DNA is transcribed into RNA [2]. The human genome project (HGP) indicates that only $2 \%$ genomic DNA is translated into protein [3], which reveals the existence of numerous non-coding RNAs. In these years' research, short non-coding RNAs such as microRNAs (miRNAs), small interfering RNAs (siRNAs) and snoRNAs have been extensively studied. Meanwhile, LncRNAs are receiving growing concerns. More and more evidences have shown that LncRNAs are not simply considered to emerge as by-products of genomes, but have plenty of definite cellular functions, many of which are connected with human diseases.
\end{abstract}


LncRNAs are generally defined to be longer than 200 nucleotides and have no open reading frame that can be translated into protein. After analyzing genome tiling arrays and high-throughput sequencing of transcriptomes, numerous LncRNAs have been discovered. In these analyses, LncRNAs have been discovered to have complicated structures and origins, so researchers consider that they shall not be purely defined based on their length and non-coding. Research suggests that LncRNAs have some common characteristics as follows. (1) Coding LncRNAs are similar to genes of coding proteins in terms of chromatin states like H3K4me3 of promoters and H3K36me3 of transcribed regions [4]. (2) The expression of LncRNAs is regulated by multiple types of common transcription factors [5]. (3) Like coding genes, LncRNAs are transcribed with RNA polymerase II, generally spliced through spliceosomes and have poly A tails. According to the position of their DNA fragments in genomes, coding LncRNAs may be divided into five categories, including sense, antisense, bidirectional, intronic and intergenic LncRNAs. The position is more of less related to the functions of these genes.

In recent years, LncRNAs have drawn an increased attention because of their functions in the human diseases including cancers. They are involved in diverse biological processes such as cell proliferation, diferentiation, chromosome remodeling, epigenetic modulation, transcriptional and posttranscriptional modifcations $[6,7]$.

\section{Roles of LncRNAs}

Some reports have claimed that more than 8000 types of LncRNAs have been discovered after a complete analysis of human genomes [8]. Some others have suggested that over 1000 types of LncRNAs are expressed in human beings and other mammals $[4,9]$. In a word, there is a large amount of LncRNAs. However, the transcripts of several types of LncRNAs are not conservative among specimens with similar genetic relationships, and only over 200 types of LncRNAs have been investigated relatively clearly at present [10]. As a result, people question if all LncRNAs have biochemical functions, and to answer this question, further research shall be conducted.

The expression of most LncRNAs is tissue-specific. Among more than 200 types of LncRNAs which have been explored relatively clearly at present, many of them have been found to have functions in vitro, whereas only some of them have been demonstrated to function in vivo. It may be judged from current data that LncRNAs get involved in extensive biological and physiological processes, having distinct functions in different stages of these processes. Furthermore, LncRNAs, as crucial regulators for promoting or inhibiting tumor development, have been discovered to play their regulatory roles from the perspectives of epigenetic, transcriptional and post-transcriptional levels. In view of their roles in development of BC, LncRNAs are classified into two types: (1) some LncRNAs that promote the development of BC and (2) some others that inhibit the development of BC. No matter they promote or inhibit the development of $\mathrm{BC}$, their mechanism of action generally covers several aspects as follows: (1) affect proliferation and apoptosis of BC cells, (2) influence drug resistance of BC cells, (3) impact invasion of $\mathrm{BC}$ cells.

\section{LncRNAs that promote development of breast cancer}

There is a range of LncRNAs that promote development of $\mathrm{BC}$, and their functions have been preliminarily investigated. It is helpful for developing more effective means for diagnosing $\mathrm{BC}$, judging its prognosis, predicting its genesis and intervening with the treatment. Hereunder, mechanisms related to these LncRNAs will be particularly introduced (see Fig. 1).

\section{Promoting proliferation of breast cancer cells or inhibiting apoptosis of breast cancer cells H19}

H19 is one of the first discovered LncRNAs, and located at the downstream of the sense of IGF2 in human genome. In multiple types of cancer, including $\mathrm{BC}$, the expression of H19 is upregulated [11]. Compared with normal mammary tissues, the expression of H19 is higher in $72.5 \%$ of $\mathrm{BC}$ [12]. $\mathrm{H} 19$ may promote proliferation of $\mathrm{BC}$ cells when they are overexpressed in these cells. As a transcription factor, E2F1 may bind with promoters of H19 to enhance its expression and thereby lead to cell proliferation [13]. In addition, the overexpression of H19 in MDA-MB-231 contributes to the colony-forming efficiency and tumorigenic abilities in vivo [14]. In $\mathrm{BC}$, the

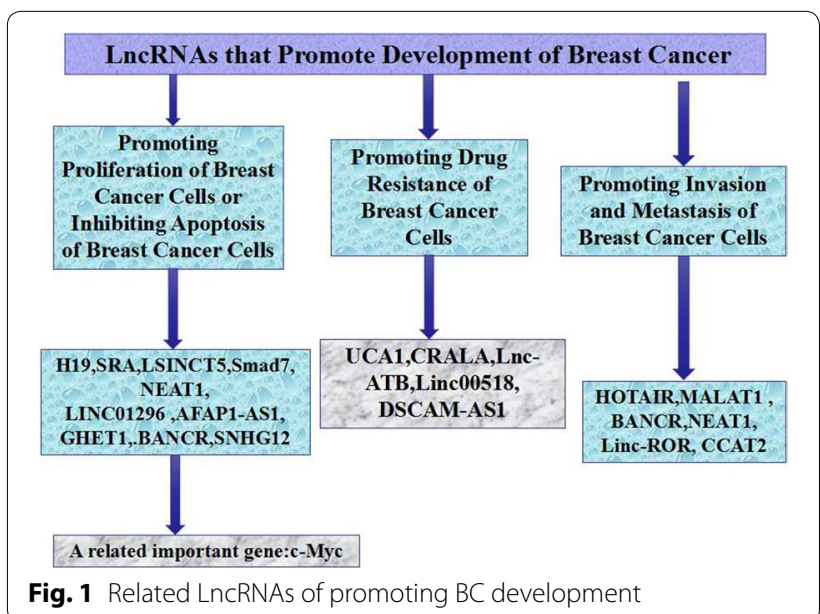


expression of $\mathrm{H} 19$ is higher in Estrogen receptor (ER $\alpha)$ positive cells, but in the ER $\alpha$ negative MDA-MB-231 cell line, ectopic overexpression of H19 is associated with increased proliferation [15]. It is clear that H19 is a category of LncRNAs that promote proliferation of $\mathrm{BC}$ cells.

\section{Steroid receptor RNA activator (SRA)}

Steroid receptor RNA activator (SRA) sites may be transcribed into encoded mRNAs or non-coding LncRNAs. It is the first LncRNA that was discovered to be not impacted by epigenetic regulation or catalytic regulation of enzymes. Selectively impacted by steroid receptors, it is a gene that gets involved in transactivating dependence of steroid receptors [16]. SRR is found to have higher expression in $\mathrm{BC}$ tissues and serum [17]. However, it has been discovered in animal experiments that pure excess SRA is inadequate for causing BC. This indicates that SRA promotes $\mathrm{BC}$, but can't lead to BC unless it acts together with other carcinogenic factors.

\section{LSINCT5}

As a type of about $2.6 \mathrm{~kb}$ long stress LncRNA, LSINCT5 is generally located in nucleus, and its genes are transcribed by RNA polymerase III instead of RNA polymerase II $[18,19]$. LSINCT5 is overexpressed in several types of $\mathrm{BC}$ cells, so is it in $\mathrm{BC}$ tissues. The proliferation of $\mathrm{BC}$ cells will be reduced if LSINCT5 is knocked out of them [19]. It is thus clear that LSINCT5 is effective for increasing proliferation of $\mathrm{BC}$ cells, so it is deemed to promote the genesis of $\mathrm{BC}$. It will be helpful for developing related reagents and drugs for diagnosing and intervening with $\mathrm{BC}$ by intensively exploring LSINCT5.

\section{Smad7}

As a type of genes located near Smad7 in mice, LncRNASmad7 has been reported to inhibit apoptosis of cancer cells with its expression in mammary epithelial cells and $\mathrm{BC}$ cell lines [20]. The anti-apoptotic functions of TGF- $\beta$ may be mediated by suppressing the expression of LncRNA-Smad7. In contrast, ectopic expression of LncRNA-Smad7 may offset apoptosis induced by TGF- $\beta$ receptor inhibitor. Nonetheless, LncRNA-Smad7 possibly only promotes the genesis of $\mathrm{BC}$ by affecting the apoptosis, because the knockout of its expression imposes no impacts upon TGF- $\beta$ induced epithelial-mesenchymal transition, Smad2 phosphorylation or expression of Smad7. This indicates that LncRNA-Smad7 is a promoter of $\mathrm{BC}$, but unlike other promoters, it merely inhibits apoptosis, so there would be new findings if it is further studied.

\section{NEAT1}

The human nuclear enriched abundant transcript 1 (NEAT1) gene encodes two LncRNA isoforms that play a central role in nuclear paraspeckles, which function in regulating RNA splicing and transcription. NEAT1 has been reported to play a critical role in mouse mammary gland development. NEAT1 functions as an oncogenic factor in multiple types of cancer, including $\mathrm{BC}$, and its expression is under the regulation of ER $\alpha$ signaling, the miR-449b-5p/c-Met axis, and hypoxia responses [21]. Studies have demonstrated that LncRNA NEAT1 promotes cancer progression. NEAT1 promoted invasion through inducing epithelial-mesenchymal transition (EMT) and NEAT1 played a role in 5-fluorouracil (5-FU) resistance. LncRNA NEAT1 could be a new diagnostic biomarker and therapy target for $\mathrm{BC}[22]$.

The meta-analysis by Yang et al. [23] suggest that there was significant difference in the OS between high NEAT1 expression level group and low NEAT1 expression level group. A significantly shorter OS was shown in the patients with high NEAT1 expression level than that with low NEAT1 expression level. Thus, it is implied that the increased expression level of NEAT1 was associated with poor OS. The meta-analysis results suggest the prognostic role NEAT1 in prognosis in the patients with different types of cancer. However, due to several limitations of the included studies, largersample size, multi-center and higher-quality studies with consistent criteria for defining high NEAT1 expression level and low NEAT1 expression level may be required to further confirm the current findings in this study. Above all, all aforementioned types of LncRNAs promote proliferation and inhibit apoptosis. It is favorable for understanding other similar LncRNAs by acquiring certain knowledge about these types of LncRNAs. Meanwhile, it will be helpful for developing pertinent means for diagnosing and intervening with $\mathrm{BC}$ by intensively investigating these genes.

\section{LINC01296}

LINC01296 exerts a tumor-promoting function in many cancers, in the regulation of proliferation and metastasis [24]. LINC01296 is up-regulated in both BC tissue samples and cells. Up-regulated LINC01296 is correlated with larger tumor size, positive lymph node metastasis, and advanced TNM stage of patients with BC. Additionally, Cox regression analysis confirmed LINC01296 as an independent prognostic indicator for patients with BC. LINC01296 may function as a potential prognostic predictor and therapeutic target for patients with $\mathrm{BC}[25]$. 


\section{AFAP1-AS1}

LncRNA actin filament-associated protein 1 antisense RNA 1 (AFAP1-AS1) is a newly recognized cancerrelated lncRNA deriving from the antisense strand of DNA at the AFAP1 coding gene locus. A slew of new studies suggest that AFAP1-AS1 is involved in many kinds of malignant tumors. Evidence has increasingly shown that AFAP1-AS1 could probably serve as a novel potential molecular biomarker in tumor diagnosis and therapeutic target in tumor treatment. A series of studies provide detailed information to understand lncRNA AFAP1-AS1 role in various human cancers. LncRNA AFAP1-AS1 is an oncogene in tumors that have been studied so far, and it may act as a useful tumor biomarker and therapeutic target [26]. The expression of AFAP1-AS1 was up-regulated in human BC tissue and associated with malignancy status, high expression of AFAP1-AS1 had a poor prognosis in BC patients. Upregulated lncRNA AFAP1-AS1 indicates a poor prognosis in $\mathrm{BC}$ patients [27].

\section{GHET1}

Song et al's study for the first time revealed the biological functions of lncRNA gastric carcinoma highly expressed transcript 1 (GHET1) in BC. Their results demonstrated that GHET1 was up-regulated in BC tissues and cell lines, and promoted BC cell proliferation, invasion and migration by affecting EMT. Their research demonstrated that GHET1 was up-regulated in $\mathrm{BC}$ tissues and cell lines, and promoted $\mathrm{BC}$ cell proliferation, invasion and migration by affecting EMT [28].

\section{BANCR}

The role of the LncRNA BRAF-regulated LncRNA 1 (BANCR) in BC has not yet been elucidated. The present study revealed that BANCR was overexpressed in $\mathrm{BC}$ cell lines and tissues, and could promote the clinical progression of disease, including increases in tumor size, lymph node metastasis and tumor-node-metastasis stage. BANCR overexpression could promote the clinical progression, metastasis and proliferation of $\mathrm{BC}$ and indicate poor prognosis of patients with BC. BANCR may therefore be a potential prognostic marker and therapeutic target of patients with BC [29]. BRAF-activated nonprotein coding RNA (BANCR) is a novel and potential regulator of cancer cell proliferation and migration [30]. LncRNA BANCR is highly expressed in BC, which is significantly correlated with the prognosis of patients. The down-regulation of BANCR can inhibit the proliferation, invasion and metastasis capacities of MCF-7 cells [31].

\section{SNHG12}

The long non-coding RNA (lncRNA) small nucleolar RNA host gene 12 (SNHG12) has a role in cell proliferation and migration. SNHG12 has been shown to play a role in a variety of human cancers [32]. The expression pattern of SNHG12 in BC and its clinical significance remains unclear. SNHG12 is upregulated in Triple-negative $\mathrm{BC}(\mathrm{TNBC})$, and its high expression is significantly correlated with tumor size and lymph node metastasis. Mechanistic investigations show that SNHG12 is a direct transcriptional target of c-MYC. SNHG12 contributes to the oncogenic potential of TNBC and may be a promising therapeutic target [33].

\section{A related important gene: c-Myc}

Oncogenic c-Myc, which is located at chromosome $8 \mathrm{q} 24$, is one of the proto-oncogenic genes which is most frequently involved in human carcinogenesis [34]. Mycinduced transformation leads to the conversion from glucose to glutamine as the oxidizable substrate which is essential to maintain TCA cycle activity. c-Myc binds to the promoters and induces the expression of several crucial regulatory genes which are involved in glutaminolytic metabolism. It has been demonstrated that supra-physiological levels of Myc expression associated with oncogenic transformation are both necessary and sufficient for the induction of glutaminolysis to the excessive level that results in "glutamine addiction" specific to tumor cells.

The c-Myc mediates elevation of glutaminolysis in cancer cells. c-Myc promotes both glutamine uptake and glutamine catabolism. Because of c-Myc-mediated metabolic reprogramming, cancer cells tend to exhibit "glutamine addiction". This is a typical example of metabolic reprogramming in cancer cells with oncogene-addiction, suggesting a potential "Achilles' heel" of tumor cells that are addicted to glutamine metabolism in manner that is mediated by c-Myc [35]. The c-Myc/Max complex inhibits the ectopic differentiation of both types of artificial stem cells. Whereas c-Myc plays a fundamental role as a "double-edged sword" promoting both induced pluripotent stem cells generation and malignant transformation. Collectively, although the further research is warranted to develop the effective anti-tumor therapeutic strategy targeting Myc family, we should always catch up with the current advances in the complex functions of Myc family in highly-malignant and heterogeneous tumor cells to realize the precision medicine [36].

As a transcription factor, MYC's primary mode of transformation is through the pro-tumorigenic transcriptional dysregulation of a wide variety of processes including proliferation, cell size, apoptosis, and metabolism. 
The alternative strategies of targeting MYC-driven cancers via selective inhibition of cellular pathways, like metabolism, that may selectively kill MYC-overexpressing cells have attractive therapeutic potential [37].

In a subset of neuroendocrine breast carcinoma tissues, the catalytic activity of EZH2 increases the number of the complex composed of N-Myc, AR, and EZH2PRC2. Enhanced levels of EZH2 protein expression and EZH2 catalytic activity play a crucial role both in murine models overexpressing N-Myc and in human castrationresistant prostate cancer cells. N-Myc redirects EZH2 activity to $\mathrm{N}-\mathrm{Myc}$ target gene promoters, resulting in the transcriptional suppression, whereas EZH2 inhibition reverses $\mathrm{N}$-Myc-driven genetic regulation. Importantly, $\mathrm{N}$-Myc sensitizes tumor cells to EZH2 inhibitors both in vitro and in vivo. In addition, $\mathrm{N}$-Myc amplification rarely occurs in other lung cancer patho-histologic findings. N-Myc amplification also occurs in approximately $40 \%$ of neuroendocrine/small-cell prostate cancers, is commonly seen concurrently with amplification of the aurora kinase A gene (AURKA), and associated with poor prognosis.

Notably, downregulation of Myc interactor (NMI), a gatekeeper of epithelial phenotype, in breast tumors promotes mesenchymal, invasive and metastatic phenotype of the cancer cells. Aberrant miR-29 expression may account for reduced NMI expression in breast tumors and mesenchymal phenotype of cancer cells that promotes invasive growth. Reduction in NMI levels has a positive feedback machinery on miR-29 levels [36].

$\mathrm{N}$-myc (MYCN), a member of the Myc family of basic-helix-loop-helix-zipper (bHLHZ) transcription factors, is a central regulator of many vital cellular processes. Overexpression of N-myc has been seen in a subset of BCs and correlates with poor prognostic features. Because phosphorylation of $\mathrm{N}$-myc is directly regulated by Gsk3 $\beta$, and indirectly by upstream signaling through the PI3K/Akt/mTOR pathway, targeting PI3K/Akt/ mTOR may be an effective therapeutic approach [38]. $\mathrm{NMI}$ is an inducible protein whose expression is compromised in advanced stage breast cancer. Analysis of mRNA of NMI and miR-29 from patient derived breast cancer tumors showed a strong, inverse relationship between the expression of NMI and the miR-29. The related studies also revealed that in the absence of NMI, miR-29 expression is upregulated due to unrestricted $\mathrm{Wnt} / \beta$ catenin signaling resulting from inactivation of GSK3 $\beta$. Reduction in NMI levels has a feed-forward impact on miR-29 levels [39].

After neoadjuvant chemotherapy, $82.4 \%$ of patients showed pathologic partial response, with only 9.8\% showing pathologic complete response. In multivariate analysis, MYC immunoreactivity and high MYC gain defined as $\mathrm{MYC} /$ nucleus $\geq 5$ were significant predictor factors for pathologic partial response. MYC may have a role in chemosensitivity to $\mathrm{AC}$ and/or docetaxel drugs. The analysis of MYC amplification may help in the identification of patients that may have a better response to $\mathrm{AC}+\mathrm{T}$ treatment [40].

\section{Promoting drug resistance of breast cancer cells}

The growth of ER-positive BC is mostly dependent upon estrogen, so anti-estrogen therapies are major means for treating this type of BC in clinical practices. Nevertheless, these therapies can't completely inhibit the growth of BC cells. BCAR4 (breast cancer antiestrogen resistance 4), as a type of LncRNA connected with resistance to tamoxifen which is an endocrine drug, may be detected in $10-27 \%$ BC samples. For patients with metastatic BC who are treated by tamoxifen, relatively high level of BCAR4 mRNA is associated with high degree of tumor malignancy and short survival. Certain report has suggested that in human BC cells ZR-75-1 and MCF7, cells can proliferate without estrogen and with various antiestrogen factors owing to artificial expression of BCAR4. Besides, BCAR4-induced resistance to endocrine drugs doesn't depend upon estrogen receptors. Therefore, BCAR4 promotes $B C$ by strengthening drug resistance of $B C$ cells. As a type of highly transformed genes, it makes the growth of cancer cells not depend upon estrogen and drug-resistant to antiestrogen therapies. As a result, it contributes to the genesis of tumors in vivo. Meanwhile, some researchers consider that BCAR4-based high tumor-specific expression would be used for treating anti-estrogen $B C$ as target. The research of Godinho et al. has suggested that both ERBB2 and ERBB3 are upregulated in the $\mathrm{BC}$ cell $\mathrm{ZR}$ that expresses BCAR4. In their research, the activation of ERBB2/ERBB3 signaling pathway is considered to be the reason why BCAR4 promotes drug resistance of $\mathrm{BC}$. BCAR4-positive patients with $\mathrm{BC}$ would benefit from BCAR4-targeted therapies. Shi et al. [41] have discovered that Lnc-ATB is upregulated in $\mathrm{BC}$ cells and tissues which are resistant to trastuzumab (i.e. a type of monoclonal antibody for immunotherapies and drug for targeted therapies). It can be induced and activated by TGF- $\beta$. By competitively binding with miR-200c, it may promote the resistance to trastuzumab and induce invasion-metastasis cascade to upregulate expressions of ZEB1 and ANF-217. As a result, EMT is caused. In addition, researchers have discovered that high-level Lnc-ATB is connected with the resistance of $\mathrm{BC}$ to trastuzumab. These research findings imply that perhaps Lnc-ATP would cause EMT and resistance to trastuzumab in patients with $\mathrm{BC}$ as a downstream factor of TGF- $\beta$. Furthermore, Jiang et al. [42] have reported that LncRNA HIF1A-AS2 and AK124454 may not only 
stimulate proliferation and invasion of $\mathrm{BC}$ cells, but also play certain roles in promoting resistance of triple-negative $B C$ to paclitaxel. Possibly used as signals for recurrence of BC, HIF1A-AS2 and AK124454 can be also utilized for strengthening therapeutic effects of paclitaxel as a target.

It is thus clear that some LncRNAs may increase resistance of $\mathrm{BC}$ cells to endocrine drugs and those for targeted therapies, so as to promote the genesis of $\mathrm{BC}$.

\section{UCA1}

Recent studies reported that long non-coding RNAs (LncRNAs) might play critical roles in regulating endocrine resistance of $\mathrm{BC}$. Urothelial carcinoma-associated 1 (UCA1) is an LncRNA with an oncogenic role in $\mathrm{BC}$. Li et al's findings reveal that tamoxifen induces UCA1 upregulation in ER-positive BC cells in a HIF1 $\alpha$ dependent manner. UCA1 upregulation results in significantly enhanced tamoxifen resistance. miR-18a inhibitor reduced the sensitivity of MCF-7 cells to tamoxifen, while miR-18a mimics sensitized BT474 cells to tamoxifen. The upregulated UCA1 sponges miR-18a, which is a negative regulator of HIF1 $\alpha$. Therefore, UCA1 upregulation is further enhanced through a miR-18a-HIF $1 \alpha$ feedback loop. In addition, our data also showed that miR-18a is a modulator of tamoxifen sensitivity due to its regulative effect on cell cycle proteins [43].

\section{CRALA}

The expression levels of chemoresistance-associated long non-coding RNA (CRALA), a newly discovered long non-coding RNA, CRALA is upregulated in chemoresistant BC cell lines. Silencing of CRALA in chemoresistant $\mathrm{BC}$ cells resensitizes the cells to chemotherapy in vitro. The univariate and multivariate analysis showed that higher CRALA expression was significantly associated with poor prognosis in $\mathrm{BC}$ patients. The study findings indicate that CRALA expression may be an important biomarker for predicting the clinical response to chemotherapy and prognosis in $\mathrm{BC}$ patients. It is possible to target CRALA to reverse chemoresistance in $\mathrm{BC}$ patients [44].

\section{Lnc-ATB}

Trastuzumab resistance (TR) is leading cause of mortality in Her-2-positive BCs, and the role of TGF- $\beta$-induced epithelial-mesenchymal transition (EMT) in trastuzumab resistance is well established, but the involvement of LncRNAs in trastuzumab resistance is still unknown. Shi et al. [41] identified long noncoding RNA activated by TGF- $\beta$ (lnc-ATB) was the most remarkably upregulated LncRNA in TR SKBR-3 cells and the tissues of TR BC patients. They found that LncRNA-ATB, a mediator of
TGF- $\beta$ signaling, could predispose BC patients to EMT and trastuzumab resistance.

\section{Linc00518}

Linc00518 expression increased nearly twofold and MRP1 level elevated about 2.5-fold in BC tissues as compared to that in adjacent normal tissues. Linc00518 could act as a molecular sponge of miR-199a to repress MRP1 expression. MRP1 depletion increased the sensitivity of MCF-7/ADR cells to ADR, VCR and PTX, and this effect was attenuated following miR-199a inhibition or linc00518 overexpression. Also, linc00518 silencing increased ADR-mediated anti-tumor effect in vivo. linc00518 downregulation reduced MDR by regulating miR-199a/MRP1 axis in BC [45].

\section{DSCAM-AS1}

Ma et al. [46] investigate the influence of long noncoding RNA (LncRNA) DSCAM-AS1 on the propagation and apoptosis of tamoxifen-resistant (TR) BC cells via regulation of mircoRNA (miR)-137 and epidermal growth factor receptor pathway substrate 8 (EPS8). They think that LncRNA DSCAM-AS1 acts as a competing endogenous RNA of miR-137 and regulates EPS8 to promote cell reproduction and suppresses cell apoptosis in tamoxifenresistant (TR) BC.

\section{Promoting invasion and metastasis of breast cancer cells HOTAIR}

Gupta et al. [47] have discovered that the expression of HOTAIR (i.e. a type of LncRNA) increases to different extent in primary $\mathrm{BC}$ tissues compared with normal mammary tissues, while it is upregulated by several hundred times and even nearly 2000 times in metastatic BC tissues. However, its expression is not so consistently high in primary tumors. In primary $\mathrm{BC}$ tissues, the high expression of HOTAIR may be considered as an index of cancer metastasis and patients' survival [47, 48]. Additionally, highly expressed HOTAIR may enhance invasion of BC cells. Furthermore, high expression of HOTAIR has been discovered in in vivo experiments to more or less promote growth and spontaneous pulmonary metastasis of tumors. The knockout of HOTAIR may significantly decrease invasion of BC cells. In terms of its mechanism, it has been discovered that highly expressed HOTAIR induces site binding of PRC2 (i.e. polycomb repressive complex 2 , a type of transcriptional inhibitor) and H3K27me3 (i.e. a kind of multiplex methylated histones) on 854 loci of genes, which are mostly downregulated by HOTAIR [47]. Chisholm et al. [49] have reported that in BC tissues, the expression of HOTAIR is positively correlated to EZH2, which is a subunit of PRC2. Compared with primary $\mathrm{BC}$ tissues, the expressions of both 
HOTAIR and EZH2 are significantly upregulated in metastatic BC tissues. Meanwhile, the co-expression of these two genes has positive correlations with poor prognosis of patients. The long noncoding RNA HOTAIR (HOX transcript antisense intergenic RNA) has been reported to be a biomarker for various malignant tumors; however, its involvement in $\mathrm{BC}$ is not fully understood. Han et al.s study suggests the higher expressions of HOTAIR and EZH2 among three BC cells. Furthermore, the downregulation of HOTAIR or silencing of EZH2 was noted to inhibit the proliferation, invasion, and migration of $\mathrm{BC}$ cells, while promoting their apoptosis [50].

\section{MALAT1}

As a type of conservative LncRNAs, metastasis-associated lung adenocarcinoma transcript 1 (MALAT1) is highly expressed in multiple categories of cancer, including $\mathrm{BC}$. The in vivo and in vitro experiments have demonstrated that MALAT1 may not only promote proliferation of triple negative BC cells, cancer development and metastasis, but also has negative correlations with the survival of patients with ER-negative HER-2 positive and triple negative $\mathrm{BC}$ in terms of its expression level [51]. In MCF7 (Luminal A) and MDA-MB-231 (triple negative), which belong to two kinds of cell lines, highconcentration 17b-Estradiol (E2) may not only inhibit cell proliferation, metastasis and invasion, but also reduce the level of MALAT1. The downregulation of MALAT1 may achieve similar effects, so E2 would impact cancer cells when the level of MALAT1 is decreased [52]. It has been discovered in research on MDA-MB-231 that KDM5B regulates MDA-MB-231 as a carcinogenic gene and the downregulation of KDM5B leads to lower expression of MALAT1 while decreasing invasion of cancer cells [53]. Above research indicates that MALAT1 has the potential to become an index for judging prognosis of $\mathrm{BC}$ and possibly turn into a target for treating $\mathrm{BC}$.

The aforementioned studies suggest that both HOTAIR and MALAT1 may promote genesis and metastasis of $\mathrm{BC}$ by enhancing invasion of $\mathrm{BC}$ cells. Furthermore, MALAT1 may enhance proliferation of $\mathrm{BC}$ cells. Both LncRNAs are helpful for treating $\mathrm{BC}$ as targets for intervening with such cancer.

\section{BANCR}

The present study revealed that LncRNA BRAF-regulated LncRNA 1 (BANCR) was overexpressed in BC cell lines and tissues, and could promote the clinical progression of disease, including increases in tumor size, lymph node metastasis and tumor-node-metastasis stage. BANCR overexpression could romote the clinical progression, metastasis and proliferation of $\mathrm{BC}$ and indicate poor prognosis of patients with $B C$. BANCR may therefore be a potential prognostic marker and therapeutic target of patients with $\mathrm{BC}$ [29].

\section{NEAT1}

LncRNA NEAT1 was highly expressed in BC tissue, and the expression was also closely related to the tumor size and lymph node metastasis. Survival study also showed that the expression of lncRNA NEAT1 was closely related with prognosis of $B C$ patients. LncRNA NEAT1 may act as an oncogene in $\mathrm{BC}$, which can promote proliferation and metastasis of $\mathrm{BC}$ [54].

\section{Linc-ROR}

Hou et al. [55] have discovered that linc-ROR was upregulated in breast tumor samples, and ectopic overexpression of linc-ROR in immortalized human mammary epithelial cells induced an epithelial-to-mesenchymal transition (EMT) program. Moreover, they showed that linc-ROR enhanced BC cell migration and invasion, which was accompanied by generation of stem cell properties. Their results indicate that linc-ROR functions as an important regulator of EMT and can promote $\mathrm{BC}$ progression and metastasis through regulation of miRNAs. Potentially, the findings of the related study implicate the relevance of linc-ROR as a possible therapeutic target for aggressive and metastatic breast cancers.

\section{CCAT2}

The present study demonstrates that TAM-resistant cells show a higher level of long non-coding RNA CCAT2 expression compared with TAM-sensitive cells. Biologically, CCAT2 knockdown could inhibit proliferation and induce apoptosis in TAM-resistant cells exposed to TAM. Furthermore, knockdown of CCAT2 improves the sensitivity to TAM in TAM-resistant cells. Overall, the study results provide a novel therapeutic approach for TAM-resistant patients through depleting CCAT2 expression [56].

\section{LncRNAs that inhibit development of breast cancer}

So far, some LncRNAs that inhibit BC from development have been explored relatively clearly. They have been discovered to mainly inhibit development of BC by inhibiting proliferation or stimulating apoptosis. See Fig. 2.

\section{Inhibiting proliferation of breast cancer cells or promoting their apoptosis}

ZFAS1

Research has suggested that Zfas1, located inside mammary ducts and acinus, is expressed differently during pregnancy and breastfeeding, which has been found to be 


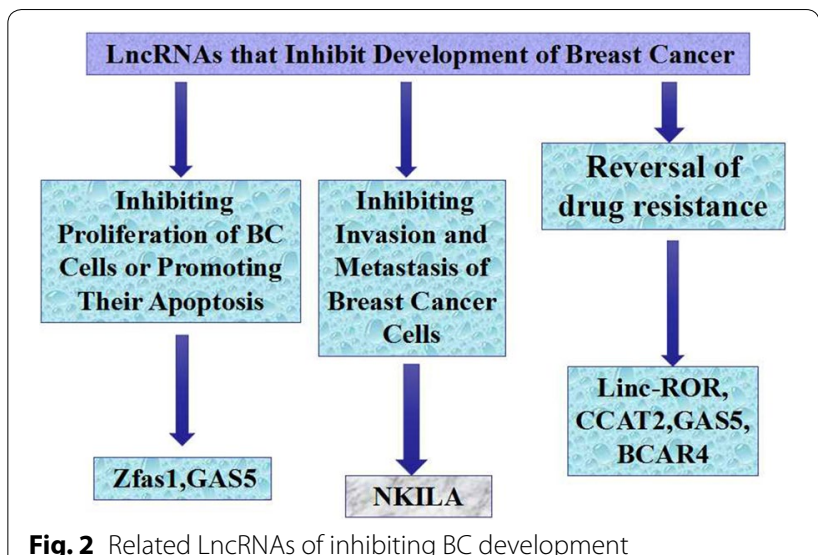

Fig. 2 Related LncRNAs of inhibiting BC development

associated with breast development. After the knockout of Zfas1 from mammary epithelial cells, proliferation of cells is strengthened. Moreover, it has been found that the expression of Zfas1 is lower in $\mathrm{BC}$ tissues than that in normal breast tissues [57]. Hence, Zfas1 is a potential inhibitor of $\mathrm{BC}$. Its functions and mechanism in the genesis of $\mathrm{BC}$ need to be further investigated, in hope of making certain contributions to developing means for intervening with and treating $\mathrm{BC}$.

\section{GAS5}

GAS5 is another LncRNA that may cause cell growth arrest and apoptosis. Compared with peripheral normal cells, LncRNA GAS5 is significantly downregulated in BC cells. Mourtada-Maarabouni et al. [58] have reported that in MCF10A and MCF7 (BC cell lines), overexpressed GAS5 may increase the apoptosis rate of these cells under UV radiation and intervention of cisplatin as an anticancer drug. In line with above research findings, Ozgur et al. [59] have reported that in apoptotic MCF7 cells under genotoxic stress, the expression of GAS5 is upregulated. In some cases, the growth arrest and apoptosis of BC cell lines can be just caused by ectopic expression of GAS5. Besides, the expression of GAS5 is high in cells under growth arrest induced by lack of nutrients or growth factors, as a result of which these cells become extremely sensitive to the stimuli promoting apoptosis [60]. It is thus clear that some LncRNAs may inhibit BC by suppressing cell proliferation and stimulating apoptosis. These LncRNAs are expected to be developed and employed for intervening with $\mathrm{BC}$.

\section{Inhibiting invasion and metastasis of breast cancer cells}

Recently, Liu et al. [61] have reported a new LncRNA known as NF-KappaB interacting LncRNA (NKILA). Upregulated by NF- $\mathrm{kB}$, it binds with NF- $\mathrm{kB} / \mathrm{I} \kappa \mathrm{B}$ to produce stable composites, so as to directly cover the phosphorylated structural domains of ІкB. As a consequence, IKK (ІкВ kinase) induced ІкB phosphorylation and NF- $\kappa B$ are activated. Importantly, NKILA may prevent excessive activation of the NF- $\mathrm{kB}$ in mammary epithelial cells under inflammatory stimuli. In MDAMB-231 (i.e. a BC cell line), NKILA may enhance apoptosis and reduce invasion. To sum up, NKILA may control invasion and metastasis of $\mathrm{BC}$ by inhibiting activation of NF-kB.

Thus, it may be inferred that some LncRNAs inhibit genesis and development of BC. In terms of its mechanism, it mainly plays its roles in inhibiting genesis and development of $\mathrm{BC}$ by suppressing proliferation of $\mathrm{BC}$ cells, or promoting apoptosis of these cells, or inhibiting invasion and metastasis of the cells. Nevertheless, no many LncRNAs have been discovered to be effective for inhibiting $\mathrm{BC}$, and very few of them have been investigated from the perspective of their inhibition mechanism. It is hoped that researchers can discover more LncRNAs that can inhibit $\mathrm{BC}$ and examine them more clearly through their efforts.

\section{Reversal of drug resistance}

Li et al's study explored the mechanism underlying long non-coding RNA ROR regulating autophagy on tamoxifen resistance in $\mathrm{BC}$. These results indicate that inhibition of long non-coding RNA ROR reverses resistance to tamoxifen by inducing autophagy in $\mathrm{BC}$ [62]. The expression levels of chemoresistance-associated long non-coding RNA (CRALA), a newly discovered long non-coding RNA, were measured by quantitative real time-PCR in 79 pre-treatment biopsied primary BC samples. Small interfering RNAs were used to knockdown CRALA expression. The effect of CRALA on chemosensitivity was evaluated using cell growth assay. The study findings indicate that CRALA expression may be an important biomarker for predicting the clinical response to chemotherapy and prognosis in $\mathrm{BC}$ patients. It is possible to target CRALA to reverse chemoresistance in $\mathrm{BC}$ patients [44]. $\mathrm{Gu}$ et al. [63] investigates the role of LncRNA growth arrest-specific transcript 5 (GAS5) in tamoxifen resistance in BC. Their study demonstrates that GAS5 enhances the efficacy of tamoxifen in the treatment of $\mathrm{BC}$ and could be a novel prognostic biomarker.

BC antiestrogen resistance 4 (BCAR4) was identified in a functional screen for genes involved in tamoxifen resistance. BCAR4 is a strong transforming gene causing estrogen-independent growth and antiestrogen resistance, and induces tumor formation in vivo. Due to its restricted expression, BCAR4 may be a good target for treating antiestrogen-resistant $\mathrm{BC}$ [64]. BCAR4 may have clinical relevance for tumour aggressiveness and 
tamoxifen resistance. The research of Godinho et al. [65] suggests that BCAR4-positive breast tumours are driven by ERBB2/ERBB3 signalling. Patients with such tumours may benefit from ERBB-targeted therapy.

\section{Use of LncRNAs-related information}

In spite of lack of complete and deep understanding about LncRNAs, people are not hindered from utilizing the conformed knowledge about these genes mainly for diagnosing $\mathrm{BC}$, judging its prognosis and predicting its metastasis and survival.

\section{LncRNAs used as diagnostic markers}

Ding et al. [66] have studied the possible applications of LncRNAs in diagnosing $\mathrm{BC}$ as a type of LncRNAs that exist among genes. They have found that lincRNA-BC2 and lincRNA-BC5 are generally upregulated by over twice in $\mathrm{BC}$ specimens compared with normal mammary tissues, whereas lincRNA-BC4 and lincRNA-BC5 are downregulated. For stage $3 \mathrm{BC}$, the expression of LincRNA-BC4 is significantly low, whereas the expression of lincRNA-BC5 is significantly high, and the expression of lincRNA-BC2 has significant positive correlations with LNM (lymph node metastasis). In particular, the expression of numerous LncRNAs is found to be highly associated with different molecular classification [67]. Furthermore, some research has suggested that LncRNAs in serum may be used for diagnosing genesis of BC. For instance, the expression of RP11-445H22.4 increases significantly in serum of patients with $\mathrm{BC}$. The sensitivity and specificity for diagnosing $\mathrm{BC}$ with this are up to $92 \%$ and $74 \%$ respectively among Chinese people [68]. Liu et al. [69] aimed to develop a long noncoding RNA (LncRNA) expression signature that can predict response to tamoxifen. A set of LncRNAs (LINC01191, RP4-639F20.1 and CTC-429P9.3) associated with distant metastasis-free survival was established. Estrogen receptor-positive $B C$ patients in the training series could be classified into high- and low-risk groups with significantly different distant metastasis-free survival values based on this signature. The LncRNA signature may have possible clinical implications in the selection of high-risk patients for tamoxifen therapy. It is thus clear that certain LncRNAs may be employed for diagnosing $\mathrm{BC}$ as biomarkers.

\section{LncRNAs used as prognostic biomarkers}

Zhao et al. [70] have discovered a range of LncRNAs which may be used for differentiating patients with $\mathrm{BC}$ from low to high risks. The high expression of LINC00324 and low expressions of PTPRG-AS1 or SNHG17 are related to the relatively longer survival. Another study has suggested that SPRY4-IT1 promotes proliferation of $\mathrm{BC}$ cells by upregulating the expression of ZNF703. In addition, increased expression of SPRY4-IT1 is connected with higher tumor volume and more severe pathological staging in patients with $\mathrm{BC}$ [71]. Hence, SPRY4-IT1 is a type of new prognostic biomarkers and potential candidates of therapeutic targets.

The overexpression of HOTAIR in BC tissues is discovered to be connected with higher invasiveness and metastasis, which implies that HOTAIR would become biomarkers for predicting overall survival. The expression of HOTAIR may independently forecast if ER-positive $\mathrm{BC}$ is metastatic. MALAT1 is discovered to have higher expression in primary $\mathrm{BC}$ and its expression further increases in the course of metastasis [72]. On the contrary, the expression of BC040587 [73], NBAT1 [74] and EGOT [75] is discovered to be downregulated in $\mathrm{BC}$ tissues, which is associated with poor prognosis. Additionally, the high expression of LINC00472 in BC tissues is connected with poor invasiveness of $\mathrm{BC}$ cells and relatively good therapeutic effects [76]. Related clinical and in vivo studies suggest that LINC00472 is a type of tumor inhibitors, so it is possibly valuable for judging prognosis and predicting therapeutic effects of $\mathrm{BC}$ in clinical practices. This suggests that some LncRNAs may be used for judging prognosis of $\mathrm{BC}$ as biomarkers and have a great prospect for application in clinical practices.

MALAT1 is a highly conserved LncRNA that is highly expressed in several types of cancer, including BC. It has been demonstrated by in vivo and in vitro studies that MALAT1 promotes proliferation, tumor development and metastasis of TNBC. In addition, the expression of MALAT1 has been reported to be negatively correlated to the survival of ER negative, lymph node negative patients of the Her-2 and TNBC molecular subtypes. It is particularly noteworthy that a recent study using genetic interventions with MALAT1 antisense nucleotides has achieved good effects for suppressing cancer development in mouse models with luminal B BC. These studies suggested that MALAT1 is expected to become a new biomarker for prognosis of $\mathrm{BCs}$ and a potential target for treating them [77].

\section{LncRNAs used for predicting drug resistance and survival as markers}

LncRNAs have potential value for being used for predicting drug resistance and survival of $\mathrm{BC}$ as markers. The overexpression of BCAR4 (a type of LncRNAs) is discovered to be effective for forecasting the resistance to tamoxifen which is a type of estrogenic drugs. On the other hand, LINC00160 and LINC01016 (i.e. two kinds of LinRNAs) are discovered to be highly expressed in ERpositive $\mathrm{BC}$ compared with ER-negative $\mathrm{BC}$ and normal tissues, which is discovered to have significant negative 
correlations with the overall survival of luminal A BC [78]. Additionally, a special BC subtype may be identified by detecting whether CCAT2 is overexpressed, having poor responses to adjuvant chemotherapies based on cyclophosphamide, methotrexate and CMF [79]. At last, the research of Chen et al. [80] has shown that the overexpression of ROR (i.e. a kind of LncRNAs) is correlated to chemotherapy resistance, which suggests that ROR may be used for predicting such resistance as an indicator. It may be inferred from the above that some LncRNAs are likely to become markers for predicting drug resistance and survival of $\mathrm{BC}$.

Therefore, researchers are exploring the applications of LncRNAs from multiple perspectives (mainly including diagnosis of $\mathrm{BC}$, judgment of prognosis, prediction of drug resistance and survival) based on their existing knowledge about them. Although it still needs some time to put them into practices, it is believed that the explorations of their applications will be more elaborate with the promotion of pertinent studies in terms of their breadth and depth. Meanwhile, new applications will be constantly developed.

\section{Conclusion}

LncRNAs belong to a type of common transcription products in genomes of human beings and mammals. Since their relationships with the genesis and development of tumors have received growing attention, the functions and mechanisms of actions of some LncRNAs have been investigated clearly in preliminary research. Nevertheless, a majority of LncRNAs remain to be further widely and deeply studied. With the improvement of research methods like the development of gene array technologies and high-throughput sequencing technologies, more categories of LncRNAs are expected to be discovered. As people acquire a deeper understanding of LncRNAs, some of these genes are likely to be developed into biomarkers for diagnosing $\mathrm{BC}$, judging and predicting its prognosis. Nevertheless, LncRNAs have been rarely reported to be used for intervening with BC. Recent CRISPR/CAS9 gene editing technologies would play certain roles in developing LncRNAs as therapies. For example, they can be employed for targeted silencing of certain LncRNAs that promote the genesis of BC.

\section{Authors' contributions}

XB provided the idea of this paper, ZX wrote this paper, other authors participated in the translation and revision of this paper. All authors contributed to the intellectual context. All authors read and approved the final manuscript.

\footnotetext{
Author details

1 Department of Surgery, Zhejiang Rehabilitation Medical Center, Hangzhou 310053, Zhejiang, China. ${ }^{2}$ Department of Breast Surgery, Zhejiang Cancer Hospital, Banshanqiao, No. 38 Guangji Road, Hangzhou 310022, Zhejiang, China. ${ }^{3}$ Department of Pathology, Zhejiang Cancer Hospital,
}

Hangzhou 310022, Zhejiang, China. ${ }^{4}$ Second Outpatient Department of Traditional Chinese Internal Medicine, Tongde Hospital of Zhejiang Province, Hangzhou 310012, Zhejiang, China.

\section{Acknowledgements \\ Not applicable.}

\section{Competing interests}

The authors declare that they have no competing interests. We claimed that this paper was original and would not have any financial interest in a company or its competitor, and that all authors meet criteria for authorship.

\section{Availability of data and materials}

This paper is a review.

\section{Consent for publication}

All authors declare that we consent for publication.

\section{Ethics approval and consent to participate}

This article does not contain any studies with human participants or animals performed by any of the authors.

\section{Funding}

This study was supported by a foundation for the " 1022 first level of innovative talents of Zhejiang Cancer Hospital, China (Grant Number: 2013102202) and Key platform technological project of Zhejiang medical science and hygiene (Grant Number: 2016ZDB003).

\section{Publisher's Note}

Springer Nature remains neutral with regard to jurisdictional claims in published maps and institutional affiliations.

Received: 2 July 2018 Accepted: 1 November 2018

Published online: 12 November 2018

\section{References}

1. Kapranov P, Cheng J, Dike S, Nix DA, Duttagupta R, Willingham AT, Stadler PF, Hertel J, Hackermüller J, Hofacker IL, Bell I, Cheung E, Drenkow J, Dumais E, Patel S, Helt G, Ganesh M, Ghosh S, Piccolboni A, Sementchenko V, Tammana H, Gingeras TR. RNA maps reveal new RNA classes and a possible function for pervasive transcription. Science. 2007:316(5830):1484-8.

2. Pennisi E. Genomics. ENCODE project writes eulogy for junk DNA. Science. 2012;337(6099):1159-61.

3. Lander ES, Linton LM, Birren B, Nusbaum C, Zody MC, Baldwin J, et al. Initial sequencing and analysis of the human genome. Nature. 2001;409(6822):860-921.

4. Guttman M, Amit I, Garber M, French C, Lin MF, Feldser D, Huarte M, Zuk O, Carey BW, Cassady JP, Cabili MN, Jaenisch R, Mikkelsen TS, Jacks T, Hacohen N, Bernstein BE, Kellis M, Regev A, Rinn JL, Lander ES. Chromatin signature reveals over a thousand highly conserved large non-coding RNAs in mammals. Nature. 2009;458(7235):223-7.

5. Yang JH, Li JH, Jiang S, Zhou H, Qu LH. ChIPBase: a database for decoding the transcriptional regulation of long non-coding RNA and microRNA genes from ChIP-Seq data. Nucleic Acids Res. 2013:41(Database issue):D177-87.

6. Tian T, Gong Z, Wang M, Hao R, Lin S, Liu K, Guan F, Xu P, Deng Y, Song D, Li N, Wu Y, Dai Z. Identification of long non-coding RNA signatures in triple-negative breast cancer. Cancer Cell Int. 2018;17(18):103.

7. Quinn JJ, Chang HY. Unique features of long non-coding RNA biogenesis and function. Nat Rev Genet. 2016;17(1):47-62.

8. Cabili MN, Trapnell C, Goff L, Koziol M, Tazon-Vega B, Regev A, Rinn $J$ L. Integrative annotation of human large intergenic noncoding RNAs reveals global properties and specific subclasses. Genes Dev. 2011;25(18):1915-27.

9. Khalil AM, Guttman M, Huarte M, Garber M, Raj A, Morales DR, Thomas $K$, Presser A, Bernstein BE, van Oudenaarden A, Regev A, Lander ES, Rinn JL. Many human large intergenic noncoding RNAs associate with 
chromatin-modifying complexes and affect gene expression. Proc Natl Acad Sci USA. 2009;106(28):11667-72.

10. Amaral PP, Clark MB, Gascoigne DK, Dinger ME, Mattick JS. IncRNAdb: a reference database for long noncoding RNAs. Nucleic Acids Res. 2011:39(Database issue):D146-51.

11. Doucrasy S, Coll J, Barrois M, Joubel A, Prost S, Dozier C, Stehelin D, Riou G. Expression of the human fetal bac h19 gene in invasive cancers. Int J Oncol. 1993;2(5):753-8.

12. Adriaenssens E, Dumont L, Lottin S, Bolle D, Leprêtre A, Delobelle A, Bouali F, Dugimont T, Coll J. Curgy JJ.H1 9 overexpression in breast adenocarcinoma stromal cells is associated with tumor values and steroid receptor status but independent of p53 and Ki-67 expression. Am J Pathol. 1998;153(5):1597-607.

13. Berteaux N, Lottin S, Monté D, Pinte S, Quatannens B, Coll J, Hondermarck $\mathrm{H}$, Curgy JJ, Dugimont T, Adriaenssens E. H19 mRNA-like noncoding RNA promotes breast cancer cell proliferation through positive control by E2F1. J Biol Chem. 2005;280(33):29625-36.

14. Lottin S, Adriaenssens E, Dupressoir T, Berteaux N, Montpellier C, Coll J, Dugimont T, Curgy JJ. Overexpression of an ectopic $\mathrm{H} 19$ gene enhances the tumorigenic properties of breast cancer cells. Carcinogenesis. 2002;23(11):1885-95.

15. Collette J, Le Bourhis $X$, Adriaenssens E. regulation of human breast cancer by the long non-coding RNA H19. Int J Mol Sci. 2017;18(11):2319.

16. Vicent GP, Nacht AS, Zaurin R, Font-Mateu J, Soronellas D, Le Dily F, Reyes $\mathrm{D}$, Beato M. Unliganded progesterone receptor-mediated targeting of an RNA-containing repressive complex silences a subset of hormoneinducible genes. Genes Dev. 2013;27(10):1179-97.

17. Lanz RB, Chua SS, Barron N, Söder BM, DeMayo F, O'Malley BW. Steroid receptor RNA activator stimulates proliferation as well as apoptosis in vivo. Mol Cell Biol. 2003;23(20):7163-76.

18. Dieci G, Fiorino G, Castelnuovo M, Teichmann M, Pagano A. The expanding RNA polymerase III transcriptome. Trends Genet. 2007;23(12):614-22.

19. Silva JM, Boczek NJ, Berres MW, Ma X, Smith DI. LSINCT5 is over expressed in breast and ovarian cancer and affects cellular proliferation. RNA Biol. 2011;8(3):496-505.

20. Arase M, Horiguchi K, Ehata S, Morikawa M, Tsutsumi S, Aburatani H, Miyazono K, Koinuma D. Transforming growth factor- $\beta$-induced IncRNASmad7 inhibits apoptosis of mouse breast cancer JygMC(A) cells. Cancer Sci. 2014;105(8):974-82.

21. Lo P-K, Zhang Y, Wolfson B, Gernapudi R, Yao Y, Duru N, Zhou Q. Dysregulation of the BRCA1/long non-coding RNA NEAT1 signaling axis contributes to breast tumorigenesis. Oncotarget. 2016;7(40):65067-89.

22. Li X, Wang S, Li Z, Long X, Guo Z, Zhang G, Zu J, Chen Y, Wen L. The InCRNA NEAT1 facilitates cell growth and invasion via the miR-211/ HMGA2 axis in breast cancer. Int J Biol Macromol. 2017;105(Pt 1):346-53.

23. Yang C, Li Z, Li Y, Xu R, Wang Y, Tian Y, Chen W. Long non-coding RNA NEAT1 overexpression is associated with poor prognosis in cancer patients:a systematic review and meta-analysis. Oncotarget. 2017;8(2):2672-80.

24. Yu X, Pang L, Yang T, Liu P. IncRNA LINC01296 regulates the proliferation, metastasis and cell cycle of osteosarcoma through cyclin D1. Oncol Rep. 2018;40(5):2507-14.

25. Jiang M, Xiao Y, Liu D, Luo N, Gao Q, Guan Y. Overexpression of long noncoding RNA LINC01296 indicates an unfavorable prognosis and promotes tumorigenesis in breast cancer. Gene. 2018;30(675):217-24.

26. Ji D, Zhong X, Jiang X, Leng K, X Y Y, Li Z, Huang L, Li J, Cui Y. The role of long non-coding RNA AFAP1-AS1 in human malignant tumors. Pathol Res Pract. 2018. https://doi.org/10.1016/j.prp.2018.08.014.

27. Ma D, Chen C, Wu J, Wang H, Wu D. Up-regulated IncRNA AFAP1-AS1 indicates a poor prognosis and promotes carcinogenesis of breast cancer. Breast Cancer. 2018;Jul:5.

28. Song R, Zhang J, Huang J, Hai T. Long non-coding RNA GHET1 promotes human breast cancer cell proliferation, invasion and migration via affecting epithelial mesenchymal transition. Cancer Biomark. 2018;22(3):565-73.

29. Jiang J, Shi SH, Li XJ, Sun L, Ge QD, Li C, Zhang W. Long non-coding RNA BRAF-regulated IncRNA 1 promotes lymph node invasion, metastasis and proliferation, and predicts poor prognosis in breast cancer. Oncol Lett. 2018;15(6):9543-52.
30. Zhang J, Du Y, Zhang X, Li M, Li X. Downregulation of BANCR promotes aggressiveness in papillary thyroid cancer via the MAPK and PI3K pathways. J Cancer. 2018;9(7):1318-28.

31. Lou KX, Li ZH, Wang P, Liu Z, Chen Y, Wang XL, Cui HX. Long noncoding RNA BANCR indicates poor prognosis for breast cancer and promotes cell proliferation and invasion. Eur Rev Med Pharmacol Sci. 2018;22(5):1358-65

32. Ruan W, Wang P, Feng S, Xue Y, Li Y. Long non-coding RNA small nucleolar RNA host gene 12 (SNHG12) promotes cell proliferation and migration by upregulating angiomotin gene expression in human osteosarcoma cells. Tumour Biol. 2016;37(3):4065-73.

33. Wang O, Yang F, Liu Y, Lv L, Ma R, Chen C, Wang J, Tan Q, Cheng Y, Xia E, Chen Y, Zhang X. C-MYC-induced upregulation of InCRNA SNHG12 regulates cell proliferation, apoptosis and migration in triple-negative breast cancer. Am J Transl Res. 2017;9(2):533-45.

34. Xu J, Chen Y, Olopade OI. MYC and breast cancer. Genes Cancer. 2010;1(6):629-40

35. Yoshida GJ. Metabolic reprogramming:the emerging concept and associated therapeutic strategies. J Exp Clin Cancer Res. 2015;34:111.

36. Yoshida GJ. Emerging roles of Myc in stem cell biology and novel tumor therapies. J Exp Clin Cancer Res. 2018;37(1):173.

37. Camarda R, Williams J, Goga A. In vivo reprogramming of cancer metabolism by MYC. Front Cell Dev Biol. 2017;5:35.

38. Beltran $\mathrm{H}$. The N-myc oncogene: maximizing its targets, regulation, and therapeutic potential. Mol Cancer Res. 2014;12(6):815-22.

39. Rostas JW, Pruitt HC, Metge BJ, Mitra A, Bailey SK, Bae S, Singh KP, Devine DJ, Dyess DL, Richards WO, Tucker JA, Shevde LA, Samant RS. microRNA-29 negatively regulates EMT regulator N-myc interactor in breast cancer. Mol Cancer. 2014;29(13):200. https://doi. org/10.1186/1476-4598-13-200.

40. Pereira CBL, Leal MF, Abdelhay ESFW, Demachki S, Assumpção PP, de Souza MC, Moreira-Nunes CA, Tanaka AMDS, Smith MC, Burbano RR. MYC amplification as a predictive factor of complete pathologic response to docetaxel-based neoadjuvant chemotherapy for breast cancer. Clin Breast Cancer. 2017;3:188-94.

41. Shi SJ, Wang LJ, Yu B, Li YH, Jin Y, Bai XZ. LncRNA-ATB promotes trastuzumab resistance and invasion-metastasis cascade in breast cancer. Oncotarget. 2015;6(13):11652-63.

42. Jiang YZ, Liu YR, Xu XE, et al. Transcriptome analysis of triple-negative breast cancer reveals an integrated mRNA-IncRNA signature with predictive and prognostic value. Cancer Res. 2016;76(8):2105-14.

43. Li X, Wu Y, Liu A, Tang X. Long non-coding RNA UCA1 enhances tamoxifen resistance in breast cancer cells through a miR-18a-HIF1a feedback regulatory loop. Tumour Biol. 2016:37(11):14733-43.

44. Li Y, Wang B, Lai H, Li S, You Q, Fang Y, Li Q, Liu Y. Long non-coding RNA CRALA is associated with poor response to chemotherapy in primary breast cancer. Thorac Cancer. 2017:8(6):582-91.

45. Chang L, Hu Z, Zhou Z, Zhang H. Linc00518 contributes to multidrug resistance through regulating the MiR-199a/MRP1 axis in breast cancer. Cell Physiol Biochem. 2018;48(1):16-28.

46. Ma Y, Bu D, Long J, Chai W, Dong J. LncRNA DSCAM-AS1 acts as a sponge of miR-137 to enhance tamoxifen resistance in breast cancer. J Cell Physiol. 2018. https://doi.org/10.1002/jcp.27105.

47. Gupta RA, Shah N, Wang KC, Kim J, Horlings HM, Wong DJ, Tsai MC, Hung T, Argani P, Rinn JL, Wang Y, Brzoska P, Kong B, Li R, West RB, van de Vijver MJ, Sukumar S, Chang HY. Long non-coding RNA HOTAIR reprograms chromatin state to promote cancer metastasis. Nature. 2010:464(7291):1071-6.

48. Sørensen KP, Thomassen M, Tan Q, Bak M, Cold S, Burton M, Larsen MJ, Kruse TA. Long non-coding RNA HOTAIR is an independent prognostic marker of metastasis in estrogen receptor-positive primary breast cancer. Breast Cancer Res Treat. 2013;142(3):529-36.

49. Chisholm KM, Wan Y, Li R, Montgomery KD, Chang HY, West RB. Detection of long non-coding RNA in archival tissue: correlation with polycomb protein expression in primary and metastatic breast carcinoma. PLoS ONE. 2012;7(10):e47998.

50. Han L, Zhang HC, Li L, Li CX, Di X, Qu X. Downregulation of long noncoding RNA HOTAIR and EZH2 induces apoptosis and inhibits proliferation, invasion, and migration of human breast cancer cells. Cancer Biother Radiopharm. 2018;33(6):241-51. 
51. Jadaliha M, Zong X, Malakar P, Ray T, Singh DK, Freier SM, Jensen T, Prasanth SG, Karni R, Ray PS, Prasanth KV. Functional and prognostic significance of long non-coding RNA MALAT1 as a metastasis driver in ER negative lymph node negative breast cancer. Oncotarget. 2016;7(26):40418-36.

52. Zhao Z, Chen C, Liu Y, Wu C. 17ß-Estradiol treatment inhibits breast cell proliferation, migration and invasion by decreasing MALAT-1 RNA level. Biochem Biophys Res Commun. 2014;445(2):388-93.

53. Bamodu OA, Huang WC, Lee WH, Wu A, Wang LS, Hsiao M, Yeh CT, Chao TY. Aberrant KDM5B expression promotes aggressive breast cancer through MALAT1 overexpression and downregulation of hsa-miR-448. BMC Cancer. 2016;16:160.

54. Zhang M, Wu WB, Wang ZW, Wang XH. IncRNA NEAT1 is closely related with progression of breast cancer via promoting proliferation and EMT. Eur Rev Med Pharmacol Sci. 2017;21(5):1020-6.

55. Hou P, Zhao Y, Li Z, Yao R, Ma M, Gao Y, Zhao L, Zhang Y, Huang B, Lu J. LincRNA-ROR induces epithelial-to-mesenchymal transition and contributes to breast cancer tumorigenesis and metastasis. Cell Death Dis. 2014;12(5):e1287.

56. Cai Y, He J, Zhang D. Suppression of long non-coding RNA CCAT2 improves tamoxifen-resistant breast cancer cells'response to tamoxifen. Mol Biol (Mosk). 2016;50(5):821-7.

57. Askarian-Amiri ME, Crawford J, French JD, Smart CE, Smith MA, Clark MB, Ru K, Mercer TR, Thompson ER, Lakhani SR, Vargas AC, Campbell IG, Brown MA, Dinger ME, Mattick JS. SNORD-host RNA Zfas1 is a regulator of mammary development and a potential marker for breast cancer. RNA. 2011;17(5):878-91.

58. Mourtada-Maarabouni M, Pickard MR, Hedge VL, Farzaneh F, Williams GT. GAS5, a non-protein-coding RNA, controls apoptosis and is downregulated in breast cancer. Oncogene. 2009;28(2):195-208.

59. Özgür E, Mert U, Isin M, Okutan M, Dalay N, Gezer U. Differential expression of long non-coding RNAs during genotoxic stress-induced apoptosis in HeLa and MCF-7 cells. Clin Exp Med. 2013;13(2):119-26.

60. Piao HL, Ma L. Non-coding RNAs as regulators of mammary development and breast cancer. J Mammary Gland Biol Neoplasia. 2012;17(1):33-42.

61. Liu B, Sun L, Liu Q, Gong C, Yao Y, Lv X, Lin L, Yao H, Su F, Li D, Zeng M, Song E. A cytoplasmic NF-kappaB interacting long noncoding RNA blocks IkappaB phosphorylation and suppresses breast cancer metastasis. Cancer Cell. 2015;27(3):370-81.

62. Li Y, Jiang B, Zhu H, Qu X, Zhao L, Tan Y, Jiang Y, Liao M, Wu X. Inhibition of long non-coding RNA ROR reverses resistance to tamoxifen by inducing autophagy in breast cancer. Tumour Biol. 2017;39(6):1010428317705790.

63. Gu J, Wang Y, Wang X, Zhou D, Shao C, Zhou M, He Z. Downregulation of IncRNA GAS5 confers tamoxifen resistance by activating miR-222 in breast cancer. Cancer Lett. 2018;10(434):1-10.

64. Godinho M, Meijer D, Setyono-Han B, Dorssers LC, van Agthoven T. Characterization of BCAR4, a novel oncogene causing endocrine resistance in human breast cancer cells. J Cell Physiol. 2011;226(7):1741-9.

65. Godinho MF, Sieuwerts AM, Look MP, Meijer D, Foekens JA, Dorssers $L C$, van Agthoven T. Relevance of BCAR4 in tamoxifen resistance and tumour aggressiveness of human breast cancer. $\mathrm{Br} J$ Cancer. 2010;12;103(8):1284-91.

66. Ding X, Zhu L, Ji T, Zhang X, Wang F, Gan S, Zhao M, Yang H. Long intergenic non-coding RNAs (LincRNAs) identified by RNA-seq in breast cancer. PLoS ONE. 2014;9(8):e103270.
67. Su X, Malouf GG, Chen Y, Zhang J, Yao H, Valero V, Weinstein JN, Spano JP, Meric-Bernstam F, Khayat D, Esteva FJ. Comprehensive analysis of long non-coding RNAs in human breast cancer clinical subtypes. Oncotarget. 2014;5(20):9864-76.

68. Xu N, Chen F, Wang F, Lu X, Wang X, Lv M, Lu C. Clinical significance of high expression of circulating serum IncRNA RP11-445H22.4 in breast cancer patients: a Chinese population-based study. Tumour Biol. 2015;36(10):7659-65.

69. Liu R, Hu R, Zhang W, Zhou HH. Long noncoding RNA signature in predicting metastasis following tamoxifen treatment for ER-positive breast cancer. Pharmacogenomics. 2018. https://doi.org/10.2217/ pgs-2018-0032.

70. Zhao W, Luo J, Jiao S. Comprehensive characterization of cancer subtype associated long non-coding RNAs and their clinical implications. Sci Rep. 2014;4:6591.

71. Shi Y, Li J, Liu Y, Ding J, Fan Y, Tian Y, Wang L, Lian Y, Wang K, Shu Y. The long noncoding RNA SPRY4-IT1 increases the proliferation of human breast cancer cells by upregulating ZNF703 expression. Mol Cancer. 2015;22(14):51.

72. Arun G, Diermeier S, Akerman M, Chang KC, Wilkinson JE, Hearn S, Kim Y, MacLeod AR, Krainer AR, Norton L, Brogi E, Egeblad M, Spector DL. Differentiation of mammary tumors and reduction in metastasis upon Malat1 IncRNA loss. Genes Dev. 2016;30(1):34-51.

73. Chi Y, Huang S, Yuan L, Liu M, Huang N, Zhou S, Zhou B, Wu J. Role of BC040587 as a predictor of poor outcome in breast cancer. Cancer Cell Int. 2014;14(1):123.

74. Hu P, Chu J, Wu Y, Sun L, Lv X, Zhu Y, Li J, Guo Q, Gong C, Liu B, Su S. NBAT1 suppresses breast cancer metastasis by regulating DKK1 via PRC2. Oncotarget. 2015;6(32):32410-25.

75. Xu SP, Zhang JF, Sui SY, Bai NX, Gao S, Zhang GW, Shi QY, You ZL, Zhan C, Pang D. Downregulation of the long noncoding RNA EGOT correlates with malignant status and poor prognosis in breast cancer. Tumour Biol. 2015;36(12):9807-12.

76. Shen Y, Katsaros D, Loo LW, Hernandez BY, Chong C, Canuto EM, Biglia $\mathrm{N}$, Lu L, Risch H, Chu WM, Yu H. Prognostic and predictive values of long non-coding RNA LINC00472 in breast cancer. Oncotarget. 2015;6(11):8579-92

77. Xiping Z, Bo C, Shifeng Y, Feijiang Y, Hongjian Y, Qihui C, Binbin T. Roles of MALAT1 in development and migration of triple negative and Her-2 positive breast cancer. Oncotarget. 2018;9(2):2255-67.

78. Jonsson P, Coarfa C, Mesmar F, Raz T, Rajapakshe K, Thompson JF, Gunaratne PH, Williams C. Single-molecule sequencing reveals estrogenregulated clinically relevant IncRNAs in breast cancer. Mol Endocrinol. 2015;29(11):1634-45.

79. Redis RS, Sieuwerts AM, Look MP, Tudoran O, Ivan C, Spizzo R, Zhang X, de Weerd V, Shimizu M, Ling H, Buiga R, Pop V, Irimie A, Fodde R, Bedrosian I, Martens JW, Foekens JA, Berindan-Neagoe I, Calin GA. CCAT2, a novel long non-coding RNA in breast cancer: expression study and clinical correlations. Oncotarget. 2013;4(10):1748-62.

80. Chen YM, Liu Y, Wei HY, Lv KZ, Fu P. Linc-ROR induces epithelial-mesenchymal transition and contributes to drug resistance and invasion of breast cancer cells. Tumour Biol. 2016;37(8):10861-70.

Ready to submit your research? Choose BMC and benefit from:

- fast, convenient online submission

- thorough peer review by experienced researchers in your field

- rapid publication on acceptance

- support for research data, including large and complex data types

- gold Open Access which fosters wider collaboration and increased citations

- maximum visibility for your research: over $100 \mathrm{M}$ website views per year

At BMC, research is always in progress.

Learn more biomedcentral.com/submissions 